\title{
Optical Correlation Using Four Wave Mixing in a Highly Nonlinear Fibre for Real-Time Serialized Ultrafast Systems
}

\author{
Fithriyah Shalihah, Arnan Mitchell, and Lam Bui
}

\begin{abstract}
A method of all-optical correlation is proposed using the nonlinear phenomena Four Wave Mixing (FWM) of two different signals. The method shows a serial solution where correlation of an ultrafast real-time serialized broadband source is demonstrated using a Highly Non-Linear Fibre (HNLF) with correlation rate reaching $200 \mathrm{MHz}$.
\end{abstract}

Keywords-broadband, Dispersive Fourier Transform, Four Wave Mixing, nonlinearity, optical correlation, Optical Signal Processing, Real-Time Ultrafast Systems

\section{INTRODUCTION}

$\mathbf{E}$ MERGING high frame rate and high resolution imaging systems have an impinging problem in that the system will be limited by the "electronic bottleneck" - the sheer amount of data to be processed by digitization is overwhelmingly large. Often, it is not the raw data that is desired, but rather the identification of the occurrence of specific events that is of primary importance. Normally, this would be achieved by digital post-processing of the recorded video, but in ultra-high bandwidth video systems this may not be practical. One possibility is to perform signal processing before digitization. Pre-processing before image acquisition circumvents the limitation imposed by the need of high-speed electronics [1], [2].

All optical image processing allows for a high degree of parallelism, where the speed of the information processed is close to the speed of light within the medium used [3]. This highly parallel all optical image processing could be applicable to cutting edge ultrafast systems such as the Serial Time Encoded Amplified Microscopy (STEAM) [4]. A novelty for systems like the STEAM is that information is encoded in the broadband spectrum of the source within a short period of time within the range of femtoseconds - allowing easily for $\mathrm{TB} / \mathrm{s}$ data rates. One of the methods used to process the spectrum encoded data is through Dispersive Fourier Transform (DFT) - frequency to time mapping of the data is undertaken simultaneously whilst the signal is amplified [5]. This time mapped signal will be used as a template input for the system proposed in this paper.

An important method of signal pre-processing is image classification and an important building block for classification is correlation. Several methods of optical signal processing

F. Shalihah is with School of Electrical and Computer Engineering RMIT, Melbourne, VIC 3000, Australia (e-mail: fithriyah.shalihah@rmit.edu.au).

A. Mitchell and L. Bui are with School of Electrical and Computer Engineering RMIT, Melbourne, VIC 3000, Australia and with ARC Centre of Excellence for Ultrahigh bandwidth Devices for Optical Systems (CUDOS). by optical correlation have previously been established using spatial components such as Spatial Light Modulators (SLMs) and Holographic elements [6], [7]. Such methods have been widely used in the applications of fingerprint matching, medical imaging, facial identification, robotics, and communication systems [8]-[10]. Although, due to the response time of the spatial elements or the download rate of the digital holographic elements, these methods can only achieve correlation rates of up to a few $\mathrm{kHz}$ [7]. This paper has demonstrated correlation rates of up to $200 \mathrm{MHz}$ speeds - a thousand times faster than the conventional correlation method. Current serialized method applicable for systems such as the STEAM is known as the Optical Time-domain Analog Image Correlator (OTAIC) [11]. This method uses Electro-Optic Modulation (EOM) namely the Mach-Zendher Modulator (MZM), in performing the multiplication function. This is very dependent on the electronic driving circuits. FWM has been used in frequency conversion in optical systems. Normally avoided in transmission systems, nonlinear processes are now largely investigated for their use in signal processing techniques [12]. The method proposed here replaces the EOM component with nonlinear optics allowing for an all optical processing method. A HNLF is chosen over a Semiconductor Optical Amplifier (SOA) in generating FWM due to the fibre's relative low loss and stability.

\section{SYSTEM CONCEPT}

The proposed method replaces the EOM component [12] with nonlinear optics allowing for an all optical multiplication in the time domain via four wave mixing (FWM). The cross

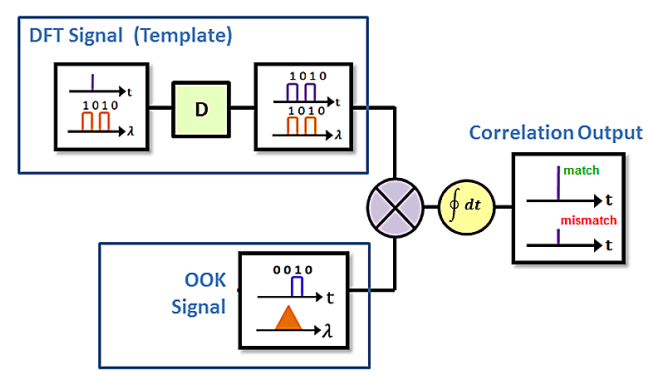

Fig. 1. Conceptual working of the proposed correlator, where the sample and template signals are multiplied then integrated to attain an overall correlation output. The template signal has a broadband encoded DFT spectrum, allowing for the correlator to be applicable in ultrafast serialized broadband real-time systems. 


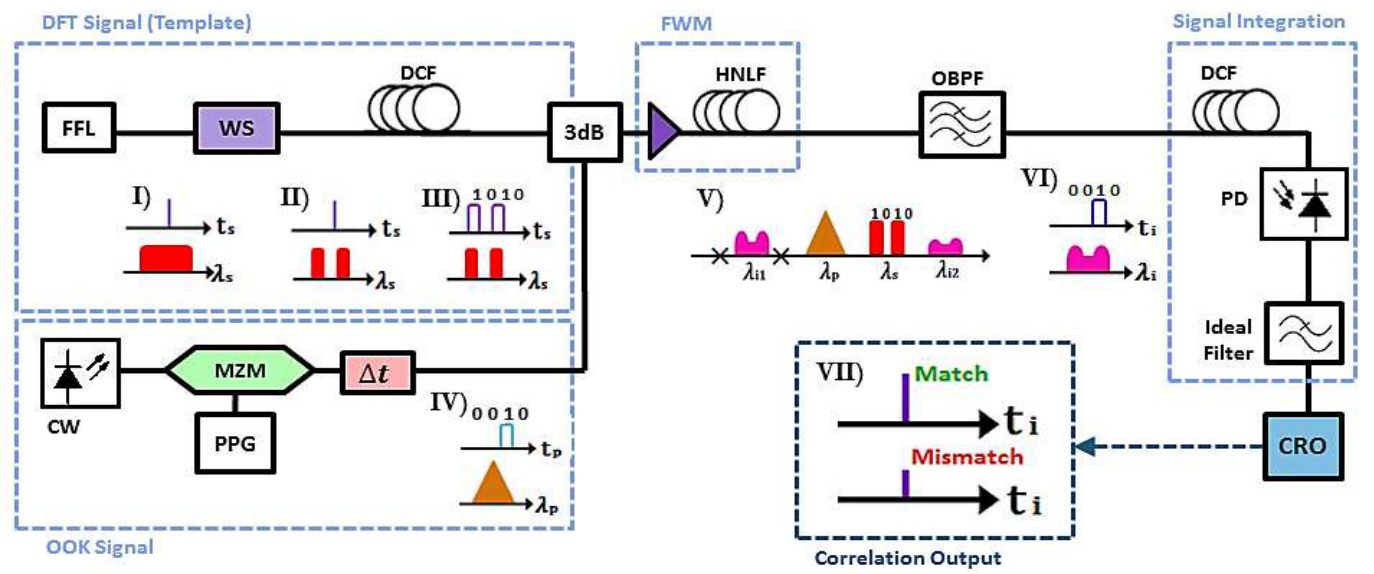

Fig. 2. System level diagram of the optical correlator using FWM, DFT signal template contains the data both in the frequency and the time domain. The reference signal uses an OOK modulation format and the nonlinear process for pattern matching using FWM is undertaken in the HNLF. The idler containing the mixing product is then filtered and integrated over another dispersive medium and further at the receiver by an ideal electronic low pass filter before digitization.

correlation function is described as [13],

$$
(S * T)(t)=\int S^{*}(\tau) T(\tau+t) d \tau
$$

where $S$ is the sample signal, $S^{*}$ is the complex conjugate of the sample signal $S$ and $T$ is the template signal. The filtered idler being the dot product of the two sources represented in time, yields the mixing product of these two signals. According to literature, FWM occurs when two frequencies of high intensity signals are combined together in phase and propagated through a highly nonlinear medium, idlers are generated. Figure 1 illustrates the system implementation.

The idlers contain the mixing product of the two signals in the time domain [14]. Thus the mixing output of two different signals in time can be predicted by characterizing the mixing process with an initial of two know inputs. Using a multiplication or an AND operation to illustrate the function in the time domain implies a convolution in the frequency domain [15], [16]. Figure 2 shows the working concept of the system, showing two arbitrary inputs. One being an ultrafast broadband time mapped template which utilizes the DFT method and the other is an On-Off Keying (OOK) modulated signal input. The two signals are then co-propagated in phase and amplified simultaneously through a Highly Non-Linear Fibre (HNLF). These signals are mixed in time through the Four Wave Mixing (FWM) process generated in the fibre. The idlers generated through the FWM process contains the mixing product in time is then optically filtered. The idler closest to the pump signal (the OOK input in this case) contains the mixing product as there is more power transfer.

The mixing product in time is then compressed using chromatic dispersion, thus integrating the multiplication into a correlation output. This correlation output is then detected electronically, where the strong peaks indicate the degree to which two bit patterns are matched. The DFT template input is a time mapped signal where data is encoded both in frequency and time. The OOK input signal is attained by modulating a Continuous Wave $(\mathrm{CW})$ laser source with a Pulsed Pattern Generator (PPG). An Electro-Optic Modulator (EOM) is used here, namely the Mach-Zendher Modulator (MZM). The FWM process takes these two high intensity broadband signals producing broadband idlers on either side of the inputs. The mixing function using the FWM phenomena is to be first characterized by using two known inputs and predicting the output by the means of a multiplication operation. The spectrum is first observed to verify the FWM process and then the time domain to verify the FWM function, an all optical correlation can then be fully realized.

\section{SYSYEM SETUP}

The system level design shown in Fig. 2 above illustrates the component set up of a photonic link simulation executed on VPI TransmissionMaker ${ }^{T M}$ software. A broadband pulsed ultrafast source of a Femtosecond Fibre Laser (FFL) is taken, where the bandwidth is $108 \mathrm{GHz}$ with pulse repetition rate of $200 \mathrm{MHz}$ and pulse width of 8 ps shown in Fig. 2-I. This is the source for the Dispersive Fourier Transform (DFT) signal template. The broadband source spectrum is then encoded with a repeating digital bit pattern using a programmable optical filter such as the WaveShaper (WS), this is shown in Fig. 2-II [17]. Each pixel or a high level bit is a filtered spectrum with a bandwidth of $22.5 \mathrm{GHz}$ and a Gaussian filter of the order 6. In this case the centre frequencies for the two high level bits or pixels are at 193.07THz and 193.09THz. The spectrum encoded signal was then mapped frequency to time via DFT (Fig. 2-III) by dispersiong through a dispersion compensating fibre (DCF) [18]. An $80.3 \mathrm{~km}$ of the DCF was used with $n=1.47$, attenuation coefficient of $0.2 \mathrm{~dB} / \mathrm{km}$ and dispersion coefficient of $-17 \mathrm{ps} /(\mathrm{km} . \mathrm{nm})$.

Figure 2-IV simply illustrates the expected output of an arbitrary bit pattern and its spectrum. The signal is a nonreturn to zero on-off keying (NRZ-OOK) modulated input. Where a CW source was used centred at $193.23 \mathrm{THz}$ with a $5 \mathrm{GHz}$ bit rate. The electro-optic modulator used was a MachZendher Modulator (MZM) with an ideal insertion loss of $0 \mathrm{~dB}$ and extinction ratio of $30 \mathrm{~dB}$. A delay line is used at $1.282 \mathrm{~ns}$ to allow for an in phase propagation between the 
two signals. The two input signals are then combined with a $3 \mathrm{~dB}$ coupler to allow for feasibility in processing broader bandwidth inputs. The two input signals now co-propagating in phase are simultaneously amplified and put through a $1 \mathrm{~km}$ long highly nonlinear fibre (HNLF) with $n=1.47$, nonlinear index of $3.2 \times 10^{-20} \mathrm{~m}^{2}$ and the attenuation and dispersion in this fibre is very low that it is negligible. The high intensity waveforms propagating in the HNLF and undergo Four Wave Mixing (FWM) as illustrated in Fig. 2-V. The idler is filtered with an ideal filter with a Gaussian order 6 with a $22.5 \mathrm{GHz}$ bandwidth at $193.36 \mathrm{THz}$ centre frequency. As per correlation function given by (1), integration is to be undertaken after signal multiplication. The integration is performed using several steps. First, group velocity dispersion (GVD) is exploited to compress the mixing product contained in the filtered idler back to a single pulse where the peak value of the pulse corresponds to the number of matching high level bits. The dispersive medium used here is an $80.01 \mathrm{~km}$ length of DCF with $n=1.47$, loss of $0.2 \mathrm{~dB} / \mathrm{km}$ and dispersion at $-17 \mathrm{ps} /(\mathrm{km} . \mathrm{nm})$. After photodetection using a PIN photodiode with an ideal responsivity of $1 \mathrm{~A} / \mathrm{W}$, the signal is then further integrated by an ideal Bessel function Low Pass Filter (LPF) with the filter order of 10 at $1.875 \mathrm{GHz}$ bandwidth.

\section{SySTEM CHARACTERIZATION}

After characterization of the system and simulating the system, the following outputs were attained. The waveform evolution of the spectrum, characterization in time and pseudorandom test of the system in time is given in Figs. 3, 4 and 5 consecutively. Figure 3 corresponds to the waveform evolution expected in the system level diagram in Fig. 2 at points III, IV, V and VI that is at the output of the frequency to time mapped template input signal, the OOK input signal, output of the HNLF and the spectrum of the filtered idler of interest correspondingly. The OOK input signal appears to show several high frequency harmonics. The

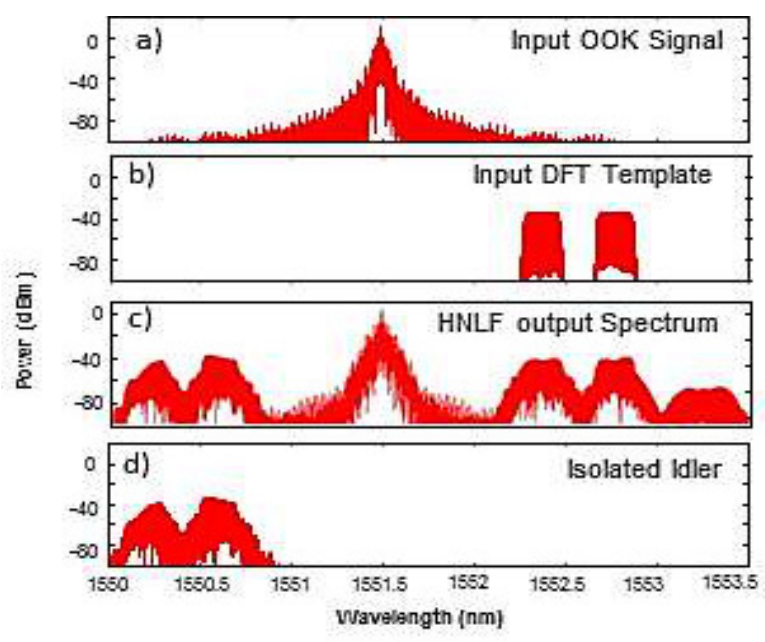

Fig. 3. Spectral Waveform evolution of the system illustrated in Fig. 2 a) is the spectrum of the OOK signal pattern, b) the ADFT signal template spectrum contains the encoded pattern 1010 , c) is the FWM result at the output of the HNLF and d) is the optically filtered idler of interest which contains the mixing product in the time domain. centre frequencies and bandwidth of the spectrum obtained and shown in Fig. 3 corresponds to the optical component settings given in Fig. 2. A characteristic of the FWM process can also be seen in Fig. 3c where the OOK input signal shown in Fig. 3a and the time mapped template input signal shown in Fig. $3 b$ is mixed and produced idlers. It can be observed that a convolution occurs in the frequency domain, such that Fig. $3 d$ is the resulting convolution of Fig. 3a and Fig. 3b, implying a multiplication in the time domain.

The temporal waveforms can be observed in Figs. 4 and 5. Where the time domain waveform evolution given in Fig. 2 is observed at points III, IV, VI and VII that is at the time mapped template input signal, the OOK input signal, filtered idler in the time domain and the electronic detection as electrical pulses correspondingly. System characterization is undertaken in the time domain where a complete match and a one bit mismatching case are considered. First a four bit pattern of 1010 for the template is chosen for simplicity.

OOK signal is the variable four bit pattern that determines the matching and mismatching condition for the system. Both the template and the OOK input signal will have the same repetition rate for each case to account for some instability that may occur in the pulses. Although, due to conservation of energy, it is expected that since the two input patterns is repeating (at $200 \mathrm{MHz}$ ) it's mixing product and its electronic correlation output pulses should also repeat at the same speed of $200 \mathrm{MHz}$ or at $5 \mathrm{~ns}$ spacing between each pulses.

Figures $4 \mathrm{a}$ to $4 \mathrm{~d}$ show the characterization of a perfect matching case where the template bit pattern is 1010 (Fig. 4b) and the OOK input signal is also 1010 (Fig. 4a). The pulse width of each pixel is $0.2 \mathrm{~ns}$ with the high level bit peak value of $7.99 \mathrm{~mW}$ for the OOK input and $1.56 \mathrm{~mW}$ for the time mapped template input signal. Using AND logic the output at the idler in time will also be 1010 (Fig. 4c) with approximately the same pulse width of $0.2 \mathrm{~ns}$ with peak pulse value of $6 \mathrm{~mW}$.

The final correlation output detected here is a Gaussian pulse at a constant peak of $0.17 \mathrm{~mA}$, FWHM of $227 \mathrm{ps}$. Due to dispersion and the photonic to electronic conversion, the final correlation output experiences pulse broadening with reference to the initial FFL pulses as well as a time shift of $2 \mathrm{~ns}$. A mismatching case is shown in Figs. $4 \mathrm{e}$ to $4 \mathrm{~h}$ having the same parameters of input with the time mapped template signal of 1010 (Fig. 4f) but with a different OOK signal pattern of 1001 (Fig. 4e). In this case there is only one high level matching bit which can be seen in the idler temporal waveform in Fig. $4 \mathrm{~g}$. Correspondingly, the correlation output expected is near half of what was attained for the matching case at peak pulse value measured as $0.11 \mathrm{~mA}$.

\section{Results}

After the system is characterized with $2^{4}=16$ possible combinations, a stream of pseudo random bit pattern (Fig. 5a) is fed through the OOK input with the same template bit pattern of 1010 (Fig. 5b). Figure 5 illustrates how a matching condition can easily be identified by the highest correlation peak attained as shown in Fig. 5d. The random bit stream still maintains a repetition rate of $200 \mathrm{MHz}$ between each consecutive pattern. The peaks shown in Fig. $5 \mathrm{~d}$ indicate the 

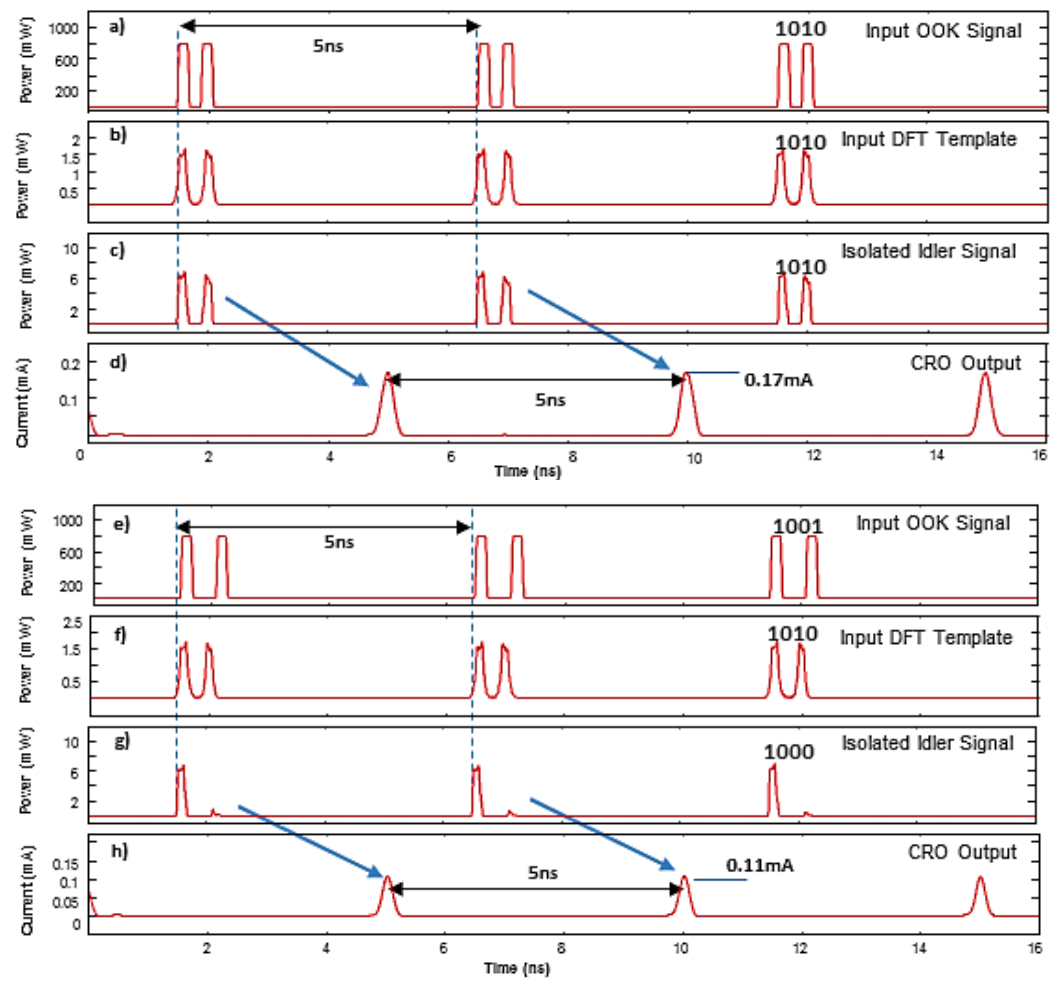

Fig. 4. Temporal Waveform for a Matching and Mismatching case. Where a) and e) is the OOK reference pattern, b) and f) is the time mapped signal of the bit pattern $1010, \mathrm{c}$ ) and g) is the idler signal showing the mixing product of waveforms a) and b) and e) and f) correspondingly. The idler signal is then compressed in time by chromatic dispersion and subsequently detected by a photodiode where the level of correlation is indicated by the peak value of the electrical Gaussian pulse shown in d) and h).

highest degree of correlation to be patterns where the high level bits matches with that of the template. These peaks correspond to the value as shown in the matching case under system characterization. Same also applies for a one high level bit mismatch.

The behaviour of the multiplicative function described in system concept is evident throughout the results given in time domain for both the characterization and the final system demonstration results. That is a matching high level bit gives a high level bit output, a matching zero does not contribute to the correlation output and a mismatching bit level gives a null. This nulling of mismatching bits allow for a good contrast at the correlation output, and are especially clear even for a four bit length pattern.

\section{DISCUSSION}

The behaviour for a matching and mismatching case is the same as expected of a multiplicative function in time, which is illustrated in characterization of the system in Fig. 4. Whilst a complementary pattern such as 0101 will give no output as shown in Fig. 5d. There is pulse broadening and time shift by 2 ns which shows consistency with characterization. From the results obtained, a perfect match of two high level bits is given at $0.17 \mathrm{~mA}$ peak and a mismatching case of one high level bit difference is $0.11 \mathrm{~mA}$ peak correlation output. There is a good contrast between a matching case and a one bit high level difference mismatch case, considering that a matching zero does not contribute to the correlation output. A complete mismatch gives a very negligible peak at the output and can be taken as a null.

A mismatching bit does not deduct from correlation output, if desired Phase Modulation (PM) approach can be taken to allow for such an operation. As previously stated, the FWM process should perform a multiplication function or much like an AND logic operation [15]. By knowing the two input patterns the mixing product at the output of the correlator can be predicted and measured, such that a perfect match of the two inputs can be seen in the time domain of the filtered idler [14]. The detected pulse at the sampling oscilloscope yields the highest correlation output in comparison to a mismatching case (Fig. 4). This was used as a reference point for when detecting for a matching pattern from a random stream of bit patterns (Fig. 5).

The results presented herein demonstrates that the FWM indeed were used to perform a multiplication function where a high level bit multiplied by another high level bit gives a high. Though, this means that any multiplication with a low level bit (null) will always give a null. Given that it does not detect for a matching null, there is room for bit error. A mismatch therefore does not subtract, hence reducing contrast. Such that a conflicting bit pattern may be taken as match as opposed to the perfect matching pattern. For example, having a template pattern of 1010 would attain the same correlation output for a 1110 as for a 1010. Current serialized correlation method for ultrafast systems, OTAIC, comparable to the proposed system uses electro-optic modulation allowing 


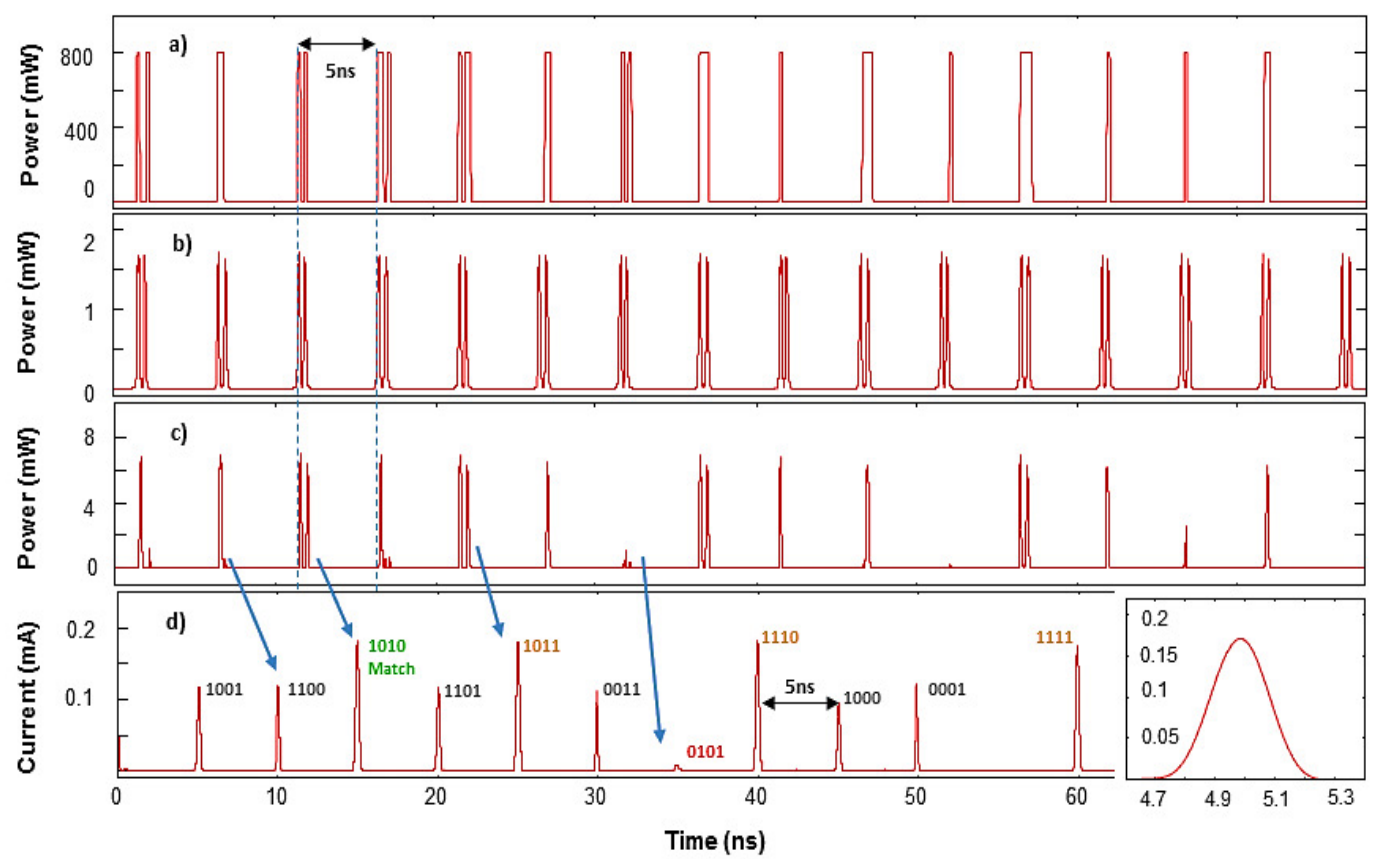

Fig. 5. Temporal waveform evolution for a series of matching cases where it can be seen clearly that a mixing output between the time mapped template signal a) and the OOK Reference Signal with pseudo random bit pattern stream b) is given in the idler signal in c). The two input signals is co-propagated in phase. The electrical waveform in d) noticeably shows where there is a matching case (green), partial mismatch (grey), conflicting patterns (orange) and complementary pattern or complete mismatch (red) - all with a time shift of $2 \mathrm{~ns}$ and maintaining a $5 \mathrm{~ns}$ spacing between each pulses (200MHz). Inset: Gaussian shaped pulses were detected from electronic detection; here the pulse shows a matching case with a peak value of $0.17 \mathrm{~mA}$.

for indication of a complimentary match to give a negative peak [12]. The OTAIC system is dependent on fast electronics as a source of reference. The demonstrated method here however allows a much greater flexibility in its inputs. Such that the two inputs can be broadband optical sources which eliminates the need for fast electronics which are very costly.

The speed of correlation is directly proportional to the repetition rate of the broadband pulsed source (from the time mapped template input). This allows for multiple frame processing and faster correlation rates means faster preprocessing. Although with faster repetition rate, pulse spacing between each consecutive pulse will reduce. This requires the pixel sizes to also reduce. In turn this implies a trade off with scalability of the bit pattern length attainable, since for shorter pulse spacing there is a shorter period for dispersion of the frequency to time mapping input, hence shorter pattern length.

An approach where the limitation of detecting for a matching zero can be further investigated by implementing an optical XNOR gate utilizing several nonlinear phenomena including FWM and cross gain modulation (XGM) [19]. According to the Boolean logic of an XNOR gate a high level bit will be generated for the matching case of both high level and low level bits highly increasing the contrast between a match and a mismatch. Current methods of optical XNOR have only been demonstrated for single tone modulated inputs. The application of an all optical logic gate using FWM in a HNLF for broadband serialized ultrafast systems shall be investigated further.

\section{CONCLUSION}

The application of signal processing in serialized broadband ultrafast systems is highly needed in pre-processing before digitization to allow for the mitigation of the problem with "electronic bottleneck". An advantage of the serialized analog time-domain process also enables robust guided-wave architecture in functional processing systems. The manipulation of the time and frequency domain also provides design guidelines to fit in much more speed out of the already existing powerful systems [20]. Reaching the fundamental limit where the processing bandwidth is comparable to the optical bandwidth within well into the $\mathrm{THz}$ range.

Traditional methods of using spatial elements have a nature of parallel processing, where an array of detector is needed which can limit greatly processing speeds. The serial approach of signal processing allows for only a single pixel photodetector to be used for electronic detection; this eliminates the need for a CCD array. FWM were used in place of the EOM component building upon the OTAIC method, addressing signal distortion and limitations of driving electronics. Utilization of the nonlinear process of FWM also allow for an all optical implementation of inputs, not limiting the system to be dependent on fast electronics. It also employs a simple design process addressing efficiency and optical bandwidth. The input and output waves in FWM process are in close proximity with respect to one another allowing easy control and filtering. It was found that by exploiting the nature of GVD an all optical correlation method can be realized in the time domain for broadband serialized ultrafast systems at an exceptionally fast correlation rate of up to $200 \mathrm{MHz}$. 


\section{ACKNOWLEDGEMENTS}

The author would like to thank the Micro-Wave Photonics and Signal Processing Group at RMIT University for their invaluable discussions. In particular Dr. Lam A. Bui for continuously providing feedback and support throughout the duration of the project, Prof. Arnan Mitchell for the invaluable guidance and encouragement, Prof. Mike Austin for his valuable discussions and advice, Mr. Paul Davies and Mr. Refat Kibria for their generous time providing insightful guidance and assistance, Mr. Jarrod Tuma and Mr. Mehrdad Shoeiby for their contributions and valuable discussions.

\section{REFERENCES}

[1] B. Borucki, "Optical Image Processing: Overview," in Visualization and Image Processing Workshop, 2007, Warsaw.

[2] M. Shoeiby, A. Mitchell, and L. A. Bui, "Novel all optical serialised real time correlator using fwm and frequency to time mapping," in 2013 International Topical Meeting on Microwave Photonics (MWP 2012), Alexandria, Virginia, USA, Oct. 2013, pp. 28-31.

[3] A. Nuruzzaman, O. Boyraz, and B. Jalali, "Time-Stretched ShortTime Fourier Transform," in IEEE Transactions on Instrumentation and Measurement, vol. 55, no. 2, 2006.

[4] K. Goda, K. K. Tsia, and B. Jalali, "Serial time-encoded amplified imaging for real-time observation of fast dynamic phenomena," Nature Letters, vol. 458, pp. 1145-1151, 2009.

[5] — , "Amplified Dispersive Fourier-Transform Imaging for Ultrafast Displacement Sensing and Barcode Reading," Applied Physics Letters, vol. 93, no. $131109,2008$.

[6] D. F. Elliott and I. L. Ayala, "Impact of sampled data on an optical joint transform correlator," Applied Optics, vol. 20, no. 11, pp. 2011-2017, 1981.

[7] N. Savage, "Digital Spatial Light Modulators," Nature Photonics, vol. 10 , no. 3, pp. 170-172, 2009.
[8] B. Javidi, J. L. Horner, and J. F. Walkup, "An Optical Pattern Recognition System for Validation and Security Verification," Optics Info Base, vol. 5, no. 9, pp. 13-18, 1994.

[9] K. Goda, A. Ayazi, D. R. Gossett, J. Sadasivam, C. K. Lonappan, E. Sollier, A. M. Fard, S. C. Hur, J. Adam, C. Murray, C. Wang, N. Brackbill, D. D. Carlo, and B. Jalali, "High-throughput singlemicroparticle imaging flow analyzer," in Proceedings of the National Academy of Sciences, USA, 2012, pp. 1-6.

[10] K. Kikuchi, "All-Optical Signal Processing Technologies in WavelengthDivision-Multiplexed Networks," Photonics Based on Wavelength Integration and Manipulation, pp. 257-270, 2005.

[11] S. H. Kim, K. Goda, A. Fard, and B. Jalali, "Optical time-domain analog pattern correlator for high-speed real-time image recognition," Optics Letters, vol. 36, no. 2, 2011.

[12] B. Javidi, P. D. Gianino, W. T. Rhodes, and B. M. Hendrickson, "Nonlinear techniques in optical pattern recognition: Introduction by the feature editors," Applied Optics, vol. 34, no. 20, pp. 3856-3857, 1995.

[13] J. H. McClellan, R. W. Schafer, and M. A. Yoder, Signal Processing First. Ney Jersey: Pearson Prentice Hall, 2003, p. 339.

[14] S. P. Singh and N. Singh, "Nonlinear Effects in Optical Fiber: Origin, Management and Applications," Progress in Electromagnetics Research, no. PIER 73, pp. 249-175, 2007.

[15] C. Thiel, "Four-wave mixing and its applications," Faculty of Washington, Washington DC, 2008.

[16] T. Acharya and A. K. Ray, Image Processing: Principles and Applications. Canada: John Wiley and Sons, Inc., 2005.

[17] F. Corporation, Programmable narrow-band filtering using the WaveShaper 1000E and 4000E. Sunnyvale, CA, 2008.

[18] D. R. S. Keisuke Goda, "Theory of amplified dispersive Fourier Transform," Physical Review A 80, 2009.

[19] S. Kumar and A. E. Willner, "Simultaneous four-wave mixing and crossgain modulation for implementing an all-optical XNOR logic gate using a single SOA," Optics Express, vol. 14, no. 12, 2006.

[20] F. Coppinger, A. S. Bhushan, and B. Jalali, "Photonic Time-Stretch and Its Applications to Analog to Digital Converter," in IEEE Transactions on Microwave Theory Technology, vol. 47, July 1999, pp. 1309-1314. 